\title{
The Proposed Development of Prototype with Secret Messages Model in Whatsapp Chat
}

\author{
Hamdani Hamdani ${ }^{1}$, Heru Ismanto ${ }^{2}$, Agus Qomaruddin Munir $^{3}$, Budi Rahmani ${ }^{4}$, Andri Syafrianto ${ }^{5}$, \\ Didit Suprihanto ${ }^{6}$, Anindita Septiarini ${ }^{7}$ \\ ${ }^{1,7}$ Department of Computer Science, Universitas Mulawarman, Samarinda, Indonesia \\ ${ }^{2}$ Department of Informatics Engineering, Universitas Musamus, Merauke, Indonesia \\ ${ }^{3}$ Department of Informatics Management, Universitas Respati Yogyakarta, Yogyakarta, Indonesia \\ ${ }^{4}$ Department of Informatics, STMIK Banjarbaru, Kalimantan Selatan, Indonesia \\ ${ }^{5}$ Department of Informatics Engineering, STMIK El Rahma, Yogyakarta, Indonesia \\ ${ }^{6}$ Department of Electronical Engineering, Universitas Mulawarman, Samarinda, Indonesia
}

\begin{tabular}{l} 
Article Info \\
\hline Article history: \\
Received May 28, 2018 \\
Revised Aug 12, 2018 \\
Accepted Aug 19, 2018 \\
\hline Keyword: \\
Chatting \\
Message \\
Secret \\
Security \\
WA \\
\hline
\end{tabular}

\begin{abstract}
Development of prototype at data security through secret messages is needed for disguising the messages sent in smartphone chatting application, WhatsApp (WA) Chat. We propose a model to disguise a plaintext message which is first encrypted by cryptosystem to change the plaintext message to ciphertext. Plaintext or plainimage entering the smartphone system is changed into encrypted text; receiver then can read the message by using similar key with the sender. The weakness of this proposal is the message random system is not planted directly in the chatting application; therefore message removing process from cryptosystem to WA application is still needed. The strength of using this model is the messages sent will not be easily re-encrypted by hacker and can be used at client computing section.
\end{abstract}

Copyright $\odot 2018$ Institute of Advanced Engineering and Science. All rights reserved.

Corresponding Author:

Hamdani Hamdani,

Departement of Computer Science,

Faculty of Computer Science and Information Technology,

Universitas Mulawarman,

Jalan Penajam, Kampus Gunung Kelua, Samarinda 75123, East Kalimantan, Indonesia.

Email: hamdani@fkti.unmul.ac.id

\section{INTRODUCTION}

It is believed that there is a flaw in the existing prototype in WhatsApp (WA) system known by hacker to re-encrypt the messages sent through our smartphone, hence additional development of the application system is needed to suit secondary security in sending and receiving secret messages through social chatting system [1]-[3].

Chatting based communication has been done by many people of smartphone users because of its effectiveness and efficiency [2], [4]-[6]. Communication by chatting is a form of the most effective textual communication used [4], e.g. the textual conversation using the smartphone by social media, chatting with WA, telegram, etc [3], [5], [7]. Writing secret message is a way needed to secure data sent through an Internet connection, thus except the sender and receiver knowing the content of the message, there will be nobody who knows and realized that there is a secret message sent in that message [8].

Plaintext and plainimage sent through chatting application are not fully secured, so a model is needed to modify the additional message in the process of sending plaintext that can be encrypted [9]. Then, there is a possibility of an attack by the hacker to re-encrypt the message sent to the receiver [10], [11]. As a consequence, the sender and receiver of the secret message are supposed to design a proposed model based 
on need and encryption method they know, so the message sent and received will be limited by encrypted text personally [12].

Chatting model done individually or in the group does not have any security guarantee to the receiver, yet the plaintext sent through operator server is fragile of hacker attack [10], [11]. Plaintext and plainimage saved in the operator server can be a weakness of the application users who feel secure but in fact there are many hacker attacks achieving textual information saved in the server [13]-[15], so an additional network control software is needed [16].

The steps of sending message through mobile is based on message demand and authentication from mobile A to mobile B [8]. This technique can use secret sharing technique using mobile SMS confirmation method as shown in Figure 1. Model shown in Figure 1 is less effective to be applied in smartphone application system because the message sent through smartphone is a long text, images and multi-participant. The example of multi-participant text sending in a chat room is shown by Figure 2.

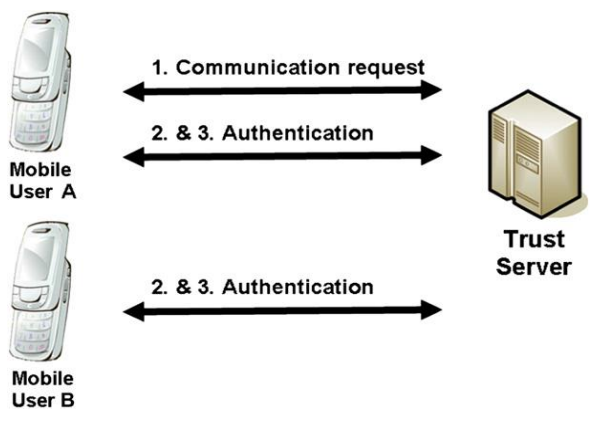

Figure 1. The authentication flowchart [8]

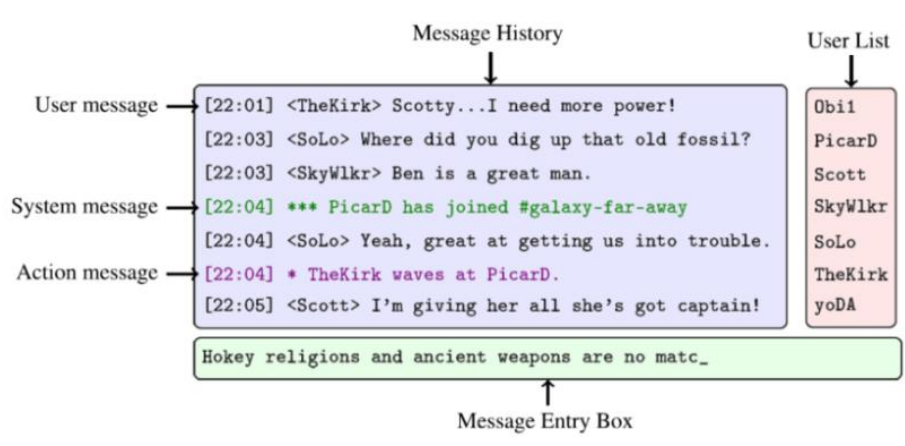

Figure 2. Abstracted chat client user interface, including examples of user, system, and action messages [5]

Figure 2 shows a chatting process in a group in a textual application. This plaintext is likely dangerous to be attacked by outsider trying to achieve those information through smartphone. In WA is already using end-to-end encryption, but we need to provide additional system outside the WA system, so users feel confident that the messages sent are not be tapped by the attackers. Next, our research focuses on how to disguise messages sent through chatting application in smartphone system used by many users nowadays. The plaintext sent will be encrypted, thus the text saved in the operator server will not be easily re-encrypted by the attackers. The text saved can be textual [17] or images [18]. Development of a model of secret messages through WA voice as in the crypt analysis of two pads in case of compressed speech [19].

\section{RELATED WORK}

In the development of the proposed model there are several related studies directly or indirectly, so some prior research is needed to produce a prototype suitable for the security needs of the next secret message. In [20] developing message authentication with a correlated setup for sending messages as done by Bob and Alice. Meanwhile, [21] analyzed the recently-proposed block cipher using cellular automata (CA) and the self-invertible function. This paper performs insecurity analysis of the cipher, because of its conjugate nature.

The results of this study also build the decryption process without knowledge of the secret key so it needs to be fixed. In [8] developing a secret-key cipher based on a non-linear structured code with the distinguishing feature that its error generator is implemented by a non-linear combination of code words of two linear complementary codes. In [2] developing a practical reputation system for pervasive social chatting with Mobile Ad Hoc Network (MANET) on smartphone. In this study [22], a general attack scenario was given in order to conduct security analyses of chaos based crypto systems with proposed cryptanalysis of a new images so it can be used in the MANET.

In the proposed algorithm, the secret key detected between sender and receiver was used in the calculation of system parameters of chaotic map group. In [1] perform a social graph based text mining framework for chat log investigation for presents a unified social graph based text mining framework. Further, it needs to be made for secure group-based mobile chat protocol [23]. For example, huge mobile 
applications (M-APPs) created for the mobile communication services which are not only for voice services, but also for social networking services. Meanwhile, [24] worked on for visual cryptography can do unscrambled by Human Visual System (HVS).

The effect of coaching and feedback on online chats in this case also found in the study [25]. Coaching occurred before each chat, and feedback was provided immediately afterwards. The findings suggest that over time, the frequency of higher-order thinking will increase more in a group that receives coaching and feedback than in a group that does not receive coaching and feedback. In addition, the findings suggest that the Community of Inquiry framework has benefits beyond its use in course design, facilitation, and assessment to include serving as a guide to coaching [25]. New steganography algorithm to conceal a large amount of secret message using hybrid adaptive neural networks with modified adaptive genetic algorithm [26]. Meanwhile, a high capacity text steganography scheme based on LZW compression and color coding [27].

The steganographic system thus embeds secret content in cover media (like text, image, audio, and video) so that its existence is not detected to an eavesdropper. Impostor Detection Through Chat Analysis also done by [3] for verify the identity of the person on the basis of his writing style. In this case we also need a model that can integrate the system in sending text. Some other research results such as in [14] and [23].

In [28] an encryption algorithm with an error detection technique and technique has been successfully verified and synthesized using Xilinx by Spartan-3E FPGA. [14] present a scheme which allows an arbitrary 2-qubit quantum state teleportation between two remote parties with control of many agents in a network, while on [23] using Mobile Chat (MC) and Mobile Chat System (MCS) for services plays a very important role in current social networking. Thus, both the Secure End-to-End Secure Mobile Chat (SE2EMC) scheme and Secure Group-based Mobile Chat (SG-MC) scheme are provided the suitable solutions. The design and implementation of secure chatting application with end to end encryption [29] and combining steganography and cryptography on android platform to achive a high-level security [30].

\section{PROPOSED METHOD}

Data security model is needed to encrypt client message before sending through the provider service, thus the message has been encrypted when it is received by the server. Plaintext and plainimage sent to the other users will not be easily read with application such as WA before decrypted to plaintext. In this method, we prepare some material consisting of cryptography model such as vigenere cipher and transposition to encrypt the message sent and to smartphone as sender and receiver.

In this case, we try to do encryption by using vigenere model, transposition and web service for testing whether the messages we sent can be encrypted in the smartphone system. Meanwhile, we use web service for integrating data from client in the smartphone so that the message written in the client application can be accepted by WA chat system. This method is proposed to secure textual message and images sent through chatting system hence the users of chatting application feel more secured. The steps of proposed model in developing prototype with secret message model as shown in Figure 3 we are working on is used for message sending process written by sender and then received by others.

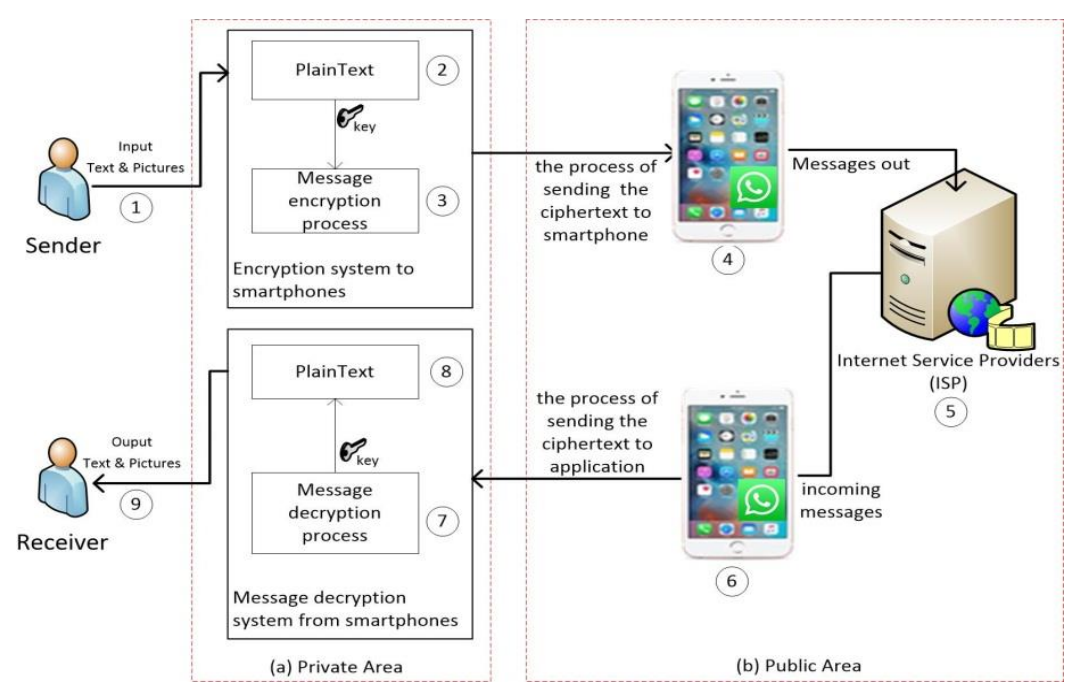

Figure 3. Proposed method of chat messages security in (a) private area to (b) public area 
In Figure 3 we can see the process in (a) private area and (b) public area in a proposed method consisting of sender (1) as the secret message sender. Sender (1) fill in the textual data or images as plaintext which is then encrypted before sending so that the message sent to the smartphone is in the form of ciphertext. The message is then sent to the providers (2) plaintext as the origin, (3) step for message encryption process (MEP) then sent to the chatting system in the smart phone (4) receive encrypted message to send to receivers through providers. (5) ISP only receive message than cannot be read the originality and then sent to the receivers as the destination (6).

The receiver (6) of encrypted message then input the encrypted message into cryptosystem in the smartphone to be encrypted (7) by message decryption process (MDP) based on private key of the sender key, thus the origin text as the plaintext (8) will be returned in the receiver system (9). Receiver (9) receives the message in the form of ciphertext which is next will be decrypted to see the original message. It can be seen that the message sent from the sender to the receiver through provider is a cryptography method encrypted message. In the proposed method we are working on, there are some methods in building prototype of secret message in chatting. They are:

\subsection{Message encryption and decryption with vigenere cipher model}

Vigenere Cipher is a polyalphabetic substitution cipher algorithm. It means, for every similar letter in a plaintext is not substituted by a letter but another letter based on the key used to encrypt. Vigenere Cipher is the simple form of polyalphabetic substitution code [31]. Vigenere Cipher is very well-known because it is very easy to understand and implement [32]. Vigenere Cipher can be seen on Figure 4.

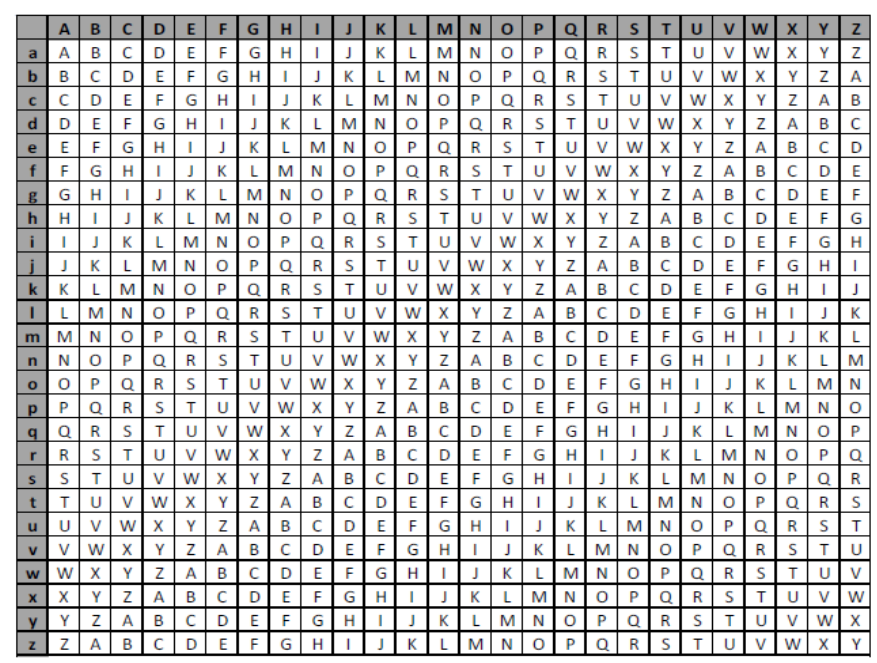

Figure 4. Vigenere cipher square method [32]

To perform Vigenere cipher encryption, draw a downward vertical line from the plaintext in the vigenere square. Then draw an across line from the key letter to the right. The letter at the intersection shows the ciphertext letter with addition [31]. Mathematically, the encryption process is expressed as follow (1):

$$
C_{i}=\left(P_{i}+K_{r}\right) \bmod 26 C
$$

Decryption is done oppositely by drawing across line from key letter to the chipertext letter, then draw a vertical line from ciphertext letter to the plaintext letter with subtraction [31]. The decryption process can be written mathematically as follow (2):

$$
C_{i}=\left(P_{i}-K_{r}\right) \bmod 26 C
$$

where,

$$
\begin{aligned}
& \left.C_{i}=\text { ciphertext (encryption text }\right) \\
& P_{i}=\text { plaintext } \\
& K_{r}=\text { key }
\end{aligned}
$$




\subsection{Message encryption and decryption with transposition model}

Transposition model is basically making ciphertext by changing plaintext object-object position without shifting the plaintext object, thus in this technique another character is not needed [33]. Reading the matrix value column by column based on the key used is done to get the ciphertext by using this transposition technique [34]. Chiper example of character transposition technique from "Universitas Mulawarman Samarinda" plaintext and using of frame 9x4 is shown by Figure 5.

\begin{tabular}{|l|c|c|c||c|c|c|}
\hline Key & 4 & 3 & 1 & 5 & 2 & 6 \\
\hline Plaint & U & N & I & V & E & R \\
\cline { 2 - 6 } & S & I & T & A & S & M \\
\cline { 2 - 6 } & U & L & A & W & A & R \\
\cline { 2 - 6 } & M & A & N & S & A & M \\
\cline { 2 - 7 } & A & R & I & N & D & A \\
\hline
\end{tabular}

(a)

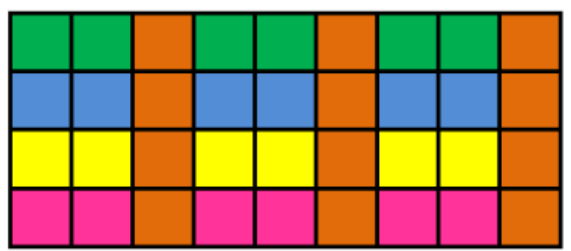

(b)

Figure 5. (a) Transposition model and (b) example of frame with 9x4

Plaintext is arranged to the right then downward by "4 31526 " key, so the cipher result will follow the key downward as "ITANI ESAAD NILAR USUMA VAWSN RMRMA". Encryption for data disguising by using key is known as encryption process. The key used is alphanumerical (a-z, A-Z, 0-9). Figure 6 shows the encryption and decryption process as shown in Figure 7 at frame 9x4 pixel.

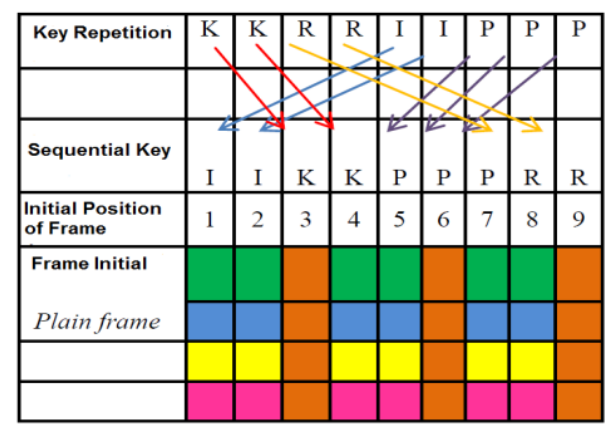

(a)

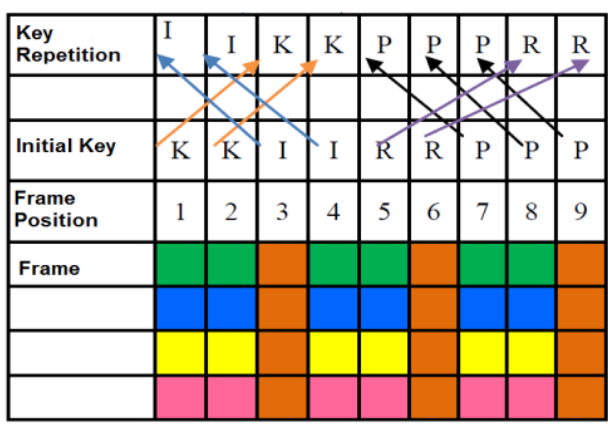

(b)

Figure 6. Process of vertical in frame (a) horizontal encryption (b) horizontal decryption

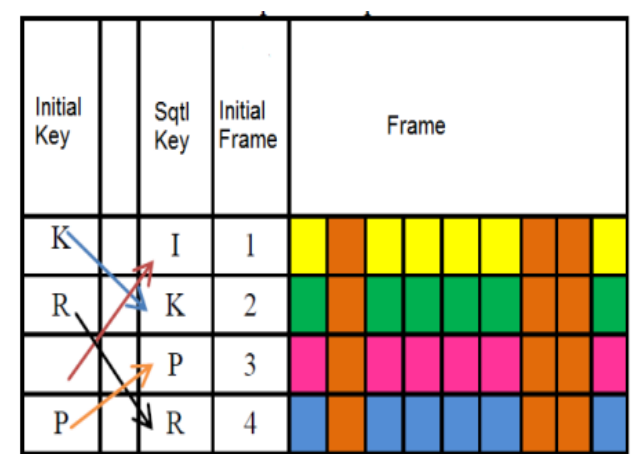

(a)

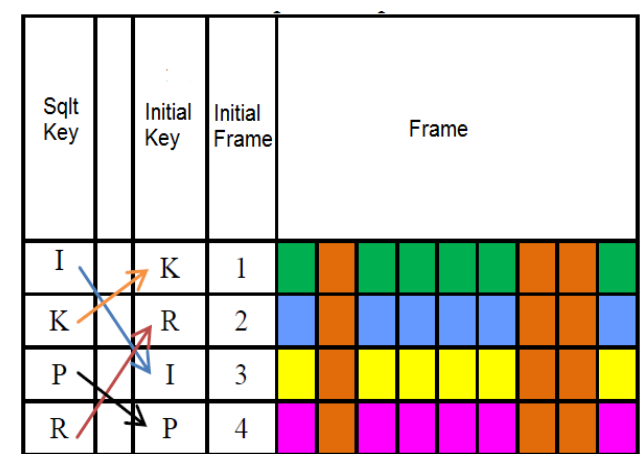

(b)

Figure 7. Process of vertical in frame (a) vertical encryption and (b) vertical decryption 
Figure 6 shows a process of encryption and decryption horizontally, meanwhile Figure 7 shows the process vertically. This model is used to jumble the number of pixel in the images so it cannot be easily recognized when it is encrypted and can be recognized when it is decrypted.

\subsection{Integration with web service model}

In this step we carry out data integration by using web service to achieve secret message through sender and receiver in which architecture is oriented in doing service producing different solution in business logic with various application [15].Web service technology combines the message sent through WhatsApp (WA) web to be disguised. The model of architecture in the web service is shown in Figure 8:

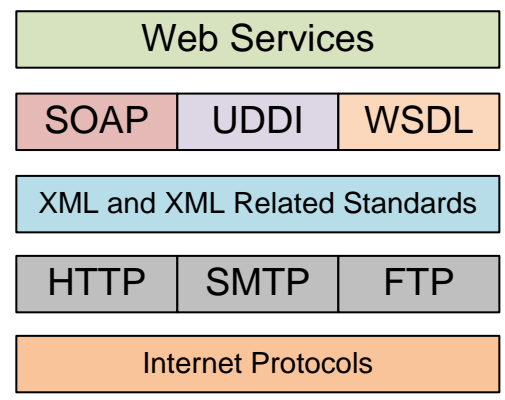

Figure 8. Web services building blocks [15]

\subsection{Autentification model}

An authentication system with a correlated setup is a pair of algorithms (MAC, VER) for a source space $S$, a message space $M$ and a distribution $P_{x y z}^{n}$ over $X^{n} \times Y^{n} \times Z^{n}$, specified as follows [20].

a. Setup $\left(1^{n}\right)$. Initially, $C$ is taken from $X^{n} \times Y^{n} \times Z^{n}$ according to $P_{x y z}^{n}$ Alice receives $X^{n}$, Bob receives $Y^{n}$ and Oscar receives $Z^{n}$.

b. Authentication $\operatorname{MAC}\left(X^{n}, S\right)$. Upon $S \in \mathrm{S}$, Alice uses her secret key $X^{n}$ to run algorithm MAC to generate a message $M=\operatorname{MAC}\left(X^{n}, S\right)$.

c. Verification $\operatorname{VER}\left(Y^{n}, M\right)$. Upon $M \in \mathrm{M}$, Bob uses his secret key $Y^{n}$ to run algorithm VER to compute $S^{-}=\operatorname{VER}(Y n, M) \in \mathrm{S} \cup\{\perp\}$, where $S^{-}=\perp$ means that Bob accepts $S^{-}$as an authenticated source state while $S^{-}=\perp$ means a reject.

\subsection{Security model}

An authentication system (MAC, VER) with a correlated setup for source space S. distribution $P_{x y z}^{n}$ $X^{n} \times Y^{n} \times Z^{n}$ is $t$-order secure if the following holds. If user attack doesn't attack, then $\operatorname{Pr}\left(S^{-}=\mathrm{S} \mid \mathrm{S}=\mathrm{S}\right)$ is $X^{n} \times Y^{n} \times Z^{n}$ is $t$-order secure if the following holds [20].

a. Authentication attack has only a negligible success probability in nin the following attack:

b. After seeing messages $M 1, \cdots, M(\leq \mathrm{t})$ from sender to receiver, attacker constructs a new message $\mathrm{M} *$ such that VER (Yn, M*) is distinct from $\perp, \mathrm{S} 1, \cdots, \mathrm{S}$, where Siis the source state in $M_{i}$.

\section{RESULTS AND DISCUSSION}

\subsection{Implementation result}

We recognize that the WA system already has end to end encryption. However, we need to propose other systems outside the system to give the WA users more confidence in the messages they send. Our research focuses on encrypting and decrypting text and images in the secret message through chatting to be applied in WA application and has successfully tested in android system application, thus the text will be first encrypted before sending to the WA application. The implementation of the secret message is shown in Figure 9.

Figure 9 shows our test results for sending secret messages into private and group chats. The result of encryption process of sentence THIS PLAINTEXT is done by using key sony sonysonys, i.e. Text for T is encrypted to key $\mathrm{S}$ and so on, as the result the step of encryption can be seen as follows: 
$(\mathrm{T}+\mathrm{S}) \bmod 26=(19+18) \bmod 26=11=\mathrm{L} \rightarrow($ applied to all text $)$

This step is applied to all letters in encryption. Ciphertext is resulted as follows:

Plaintext : : THIS PLAINTEXT

Key : : sony sonysonys

Ciphertext : LVVQ HZNGFHRVL

To find plaintext result in decryption, this step is applied:

$$
(\mathrm{L}-\mathrm{S}) \bmod 26=(11-18) \bmod 26=19=\mathrm{T} \rightarrow(\text { applied to all text })
$$

The result of message decryption can be seen as follows:

$\begin{array}{ll}\text { Ciphertext } & : \text { LVVQ HZNGFHRVL } \\ \text { Key } & : \text { sony sonysonys } \\ \text { Plaintext } & : \text { THIS PLAINTEXT }\end{array}$

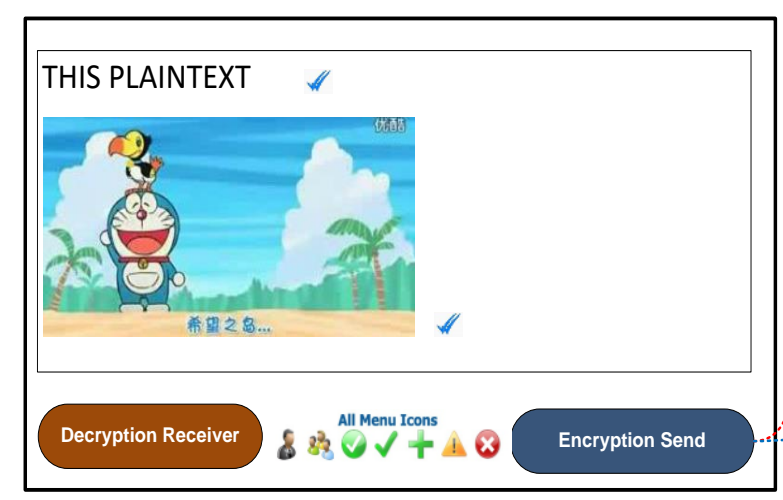

(a)

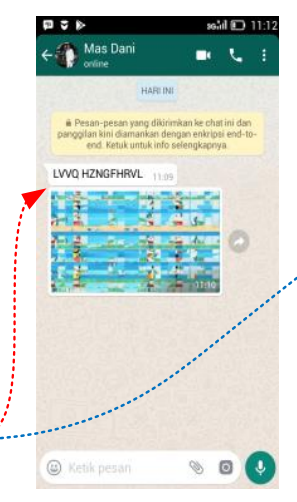

(b)

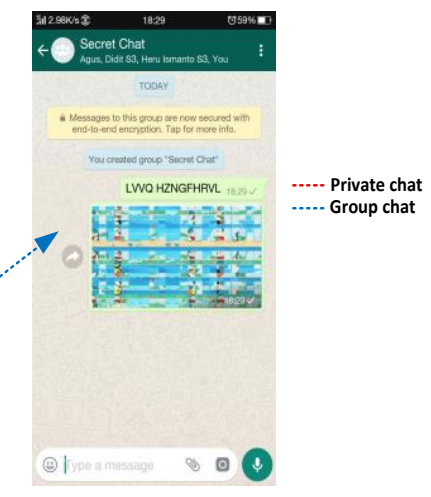

(c)

Figure 9. (a) Implementation of plain text and picture to WhatsApp in (b) private and (c) group secret chat

\subsection{Discussion result}

There are some weaknesses we need to improve in the model we are working on. For instance, how to not separate secret message system and WA so that the model developed does not need to carry out two steps of the process; message sent encryption and message received decryption in the system and sending a message to the WA system. We assume it is more practical and safe to have the model separately with the WA so that users do not need to encrypt the message they do not intend to if it does not require a secret thing.

\section{CONCLUSION}

The model developed has produced a new prototype in chatting application to add secret message as text security. The model can be used by users of the application in their smartphone. As the result, users will be more secure in using social chatting application than before. In the next research, we are trying to integrate the prototype into social chatting application in web so that the model can be used immediately and embedded in WA system.

\section{REFERENCES}

[1] T. Anwar and M. Abulaish, "A Social Graph based Text Mining Framework for Chat Log Investigation", Digit. Investig., vol. 11, no. 4, pp. 349-362, Dec. 2014.

[2] Z. Yan, et al., "A Practical Reputation System for Pervasive Social Chatting", J. Comput. Syst. Sci., vol. 79, no. 5, pp. 556-572, Aug. 2013.

[3] S. Shrivastava and P. Singh, "Impostor Detection through Chat Analysis", Procedia Comput. Sci., vol. 89, pp. 540-548, 2016. 
[4] F. A. B. H. Ali and S. M. Aydah, "Development of Prototype Chat System Using Mobile Platform for Disable People", Procedia - Soc. Behav. Sci., vol. 57, pp. 33-39, Oct. 2012.

[5] D. C. Uthus and D. W. Aha, "Multiparticipant Chat Analysis: A Survey", Artif. Intell., vol. 199-200, pp. 106-121, June 2013.

[6] E. Hatfield and R. L. Rapson, "From Pen Pals to Chat Rooms: The Impact of Social Media on Middle Eastern Society", Springerplus, vol. 4, p. 254, January 2015.

[7] T. Sutikno, et al., "WhatsApp , Viber and Telegram: Which is the Best for Instant Messaging ?," Int. J. Electr. Comput. Eng., vol. 6, no. 3, pp. 909-914, 2016.

[8] V. C. da Rocha and D. L. de Macedo, "A Secret-Key Cipher based on a Non-Linear Structured Code", Comput. Commun., vol. 22, pp. 758-761, 1999.

[9] M. Caboara, et al., "Lattice Polly Cracker cryptosystems", J. Symb. Comput., vol. 46, no. 5, pp. 534-549, May 2011.

[10] B. Purnama and a. H. H. Rohayani, “A New Modified Caesar Cipher Cryptography Method with LegibleCiphertext From a Message to Be Encrypted", Procedia Comput. Sci., vol. 59, no. Iccsci, pp. 195-204, 2015.

[11] A. M. Youssef, "Cryptanalysis of a Quadratic Knapsack cryptosystem", Comput. Math. with Appl., vol. 61, no. 4, pp. 1261-1265, Feb. 2011.

[12] S. Oukili and S. Bri, "High Throughput FPGA Implementation of Data Encryption Standard with Time Variable Sub-Keys", Int. J. Electr. Comput. Eng., vol. 6, no. 1, pp. 298-306, 2016.

[13] X. Sun, "A Scheme of Concealment and Transmission of Secret Information in Text Files", Phys. Procedia, vol. 25, pp. 1365-1368, 2012.

[14] Z. Zhang, "Controlled Teleportation of an Arbitrary n-qubit Quantum Information using Quantum Secret Sharing of Classical Message", Phys. Lett. A, vol. 352, no. 1-2, pp. 55-58, Mar. 2006.

[15] E. Moradian, "Integrating Web Services and Intelligent Agents in Supply Chain for Securing Sensitive Messages", in International Conference on Knowledge-Based and Intelligent Information and Engineering Systems, pp. 771-778, 2008.

[16] I. Alsmadi and D. Xu, "Security of Software Defined Networks: A Survey", Comput. Secur., vol. 53, pp. 79-108, September 2015.

[17] L. D. Singh and K. M. Singh, "Implementation of Text Encryption using Elliptic Curve Cryptography", Procedia Comput. Sci., vol. 54, no. 1, pp. 73-82, 2015.

[18] J. Zarepour-Ahmadabadi, et al., "An Adaptive Secret Image sharing with a new Bitwise Steganographic Property", Inf. Sci. (Ny)., vol. 369, pp. 467-480, November 2016.

[19] L. A. Khan, et al., "Crypt Analysis of Two Time Pads in Case of Compressed Speech", Comput. Electr. Eng., vol. 37, no. 4, pp. 559-569, July 2011.

[20] S. Jiang, "On message authentication with a correlated setup", Inf. Process. Lett., vol. 116, no. 4, pp. 289-293, April 2016.

[21] J. Sung, et al., "Cryptanalysis of an Involutional Block Cipher using Cellular Automata", Inf. Process. Lett., vol. 104, no. 5, pp. 183-185, Nov. 2007.

[22] F. Özkaynak and A. B. Özer, "Cryptanalysis of a new Image Encryption Algorithm based on Chaos", Opt. - Int. J. Light Electron Opt., vol. 127, no. 13, pp. 5190-5192, July 2016.

[23] H.-C. Chen, et al., "A Secure group-based Mobile Chat Protocol”, J. Ambient Intell. Humaniz. Comput., vol. 7, no. 5, pp. 693-703, Maret 2016.

[24] A. V. Dahat and P. V. Chavan, "Secret Sharing based Visual Cryptography Scheme Using CMY Color Space", Procedia Comput. Sci., vol. 78, pp. 563-570, 2016.

[25] D. S. Stein, et al., "From 'Hello' to Higher-Order Thinking: The Effect of Coaching and Feedback on Online Chats", Internet High. Educ., vol. 16, pp. 78-84, January 2013.

[26] N. N. El-Emam and R. A. S. AL-Zubidy, "New steganography algorithm to conceal a large amount of secret message using hybrid adaptive neural networks with modified adaptive genetic algorithm," J. Syst. Softw., vol. 86, no. 6, pp. 1465-1481, June 2013.

[27] A. Malik, et al., "A high Capacity Text Steganography Scheme based on LZW Compression and Color Coding", Eng. Sci. Technol. an Int. J., pp. 4-11, August 2016.

[28] T. Narendra Babu, et al., "Implementation of High Security Cryptographic System with Improved Error Correction and Detection Rate using FPGA", Int. J. Electr. Comput. Eng., vol. 6, no. 2, pp. 602-610, 2016.

[29] A. H. Ali and A. M. Sagheer, "Design and Implementation of Secure Chatting Application with end to end Encryption", J. Eng. Appl. Sci., vol. 12, no. 1, pp. 156-160, 2017.

[30] S. H. Ahmed, et al., "Combining Steganography and Cryptography on Android Platform to Achive a High-Level Security", J. Eng. Appl. Sci., vol. 12, no. 17, pp. 4448-4452, 2017.

[31] A. Bhateja and S. Kumar, "Genetic Algorithm with Elitism for Cryptanalysis of Vigen ere Cipher", in Issues and Challenges in Intelligent Computing Techniques (ICICT), 2014 International Conference on, 2014, pp. 373-377.

[32] A. Saraswat, et al., "An Extended Hybridization of Vigenere and Caesar Cipher Techniques for Secure Communication”, Procedia - Procedia Comput. Sci., vol. 92, pp. 355-360, 2016.

[33] B. H. Krishna, et al., "Multiple Text Encryption, Key Entrenched, Distributed Cipher using Pairing Functions and Transposition Ciphers", in This full-text paper was peer-reviewed and accepted to be presented at the IEEE WiSPNET 2016 conference, 2016, pp. 1059-1061.

[34] M. Jain and S. K. Lenka, "Secret Data Transmission using Vital Image Steganography over Transposition Cipher", in Green Computing and Internet of Things (ICGCIoT), 2015 International Conference on, 2016, pp. 1026-1029. 


\section{BIOGRAPHIES OF AUTHORS}
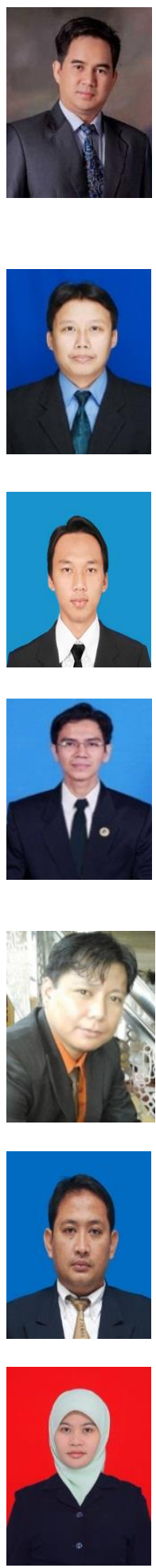

Dr. Hamdani is a lecturer and a researchers at Department of Computer Science in Universitas Mulawarman, Indonesia. He obtained his bachelor degree of Informatics in Universitas Ahmad Dahlan, Indonesia (2002). He obtained his Master of Computer Science in Universitas Gadjah Mada, Indonesia (2009) and Ph.D in Computer Science at Department of Computer Science \& Electronics in Universitas Gadjah Mada, Yogyakarta, Indonesia (2018). His research interests include decision support systems (DSS), group decision support systems (GDSS), social networks analysis, intelligent system, information security and web engineering. E-mail : hamdani@fkti.unmul.ac.id; dani@ieee.org / Web : http://odahetam.com

Heru Ismanto received his bachelor's of Informatics, and Master of Computer Science from Universitas Padjadjaran (1996) and Universitas Gadjah Mada (2009) respectively. Currently he is a lecturer at Department of Informatics Engineering, Universitas Musamus, Merauke, Papua and his a Ph.D student at Department of Computer Science and Electronics, Universitas Gadjah Mada since 2014. He was interesting in Decision Support System, GIS, e-Government System. Email: heru@unmus.ac.id

Agus Qomaruddin Munir received his bachelor's of Informatics, and Master of Computer Science from Universitas Islam Indonesia (2001) and Universitas Gadjah Mada (2009) respectively. Currently he is a lecturer at Department of Informatics Engineering, Universitas Respati Yogyaarta and his a PhD student at Department of Computer Science and Electronics, Universitas Gadjah Mada since 2014. He was interesting in Decision Support System, GIS, e-Government System. Email: agusqmnr@yahoo.com

Budi Rahmani received his bachelor's of Electrical Engineering, and Master of Informatics from Yogyakarta State University and Dian Nuswantoro University in 2003 and 2010 respectively. Currently he is a PhD student at Department of Computer Science and Electronics, Universitas Gadjah Mada since 2014. He was interesting in Embedded System and Robotics. Currently his research focused is computer vision and control system for robot. His other research interests include decision support system using artificial neural network. He can be contacted by email: budirahmani@gmail.com http://budirahmani.wordpress.com

Andri Syafrianto is a lecturer and a researches at Department of Informatics Engineering in STMIK El Rahma, Yogyakarta and Born April 9 $9^{\text {th }}, 1983$ in Palembang, Indonesia. He obtained his bachelor degree of Informatics Engineering in Universitas Bina Darma Palembang, Indonesia (2005). He obtained his Master of Computer Science in Universitas Gadjah Mada, Indonesia (2010). His research interests include decision support systems (DSS), intelligent system, Information system and Cryptanalysis. Email: andrisyafrianto@gmail.com

Didit Suprihanto Currently he is a PhD Student at Department of Computer Science and Electronics Gadjah Mada University and lecturer at Department of Electrical Engineering, Universitas Mulawarman, Samarinda, Indonesia. His research interests include computer networks security, e-Government related issues and security assessment. Email: didit.suprihanto@ft.unmul.ac.id

Dr. Anindita Septiarini was born in Nganjuk, East Java, Indonesia in September $1^{\text {st }} 1982$. She received her Bachelor degree in Informatic Engineering of Universitas Surabaya, Indonesia in 2005. She received her Master of Computer Scince degree at Universitas Gadjah Mada, Yogyakarta, Indonesia in 2009 and Ph.D in Computer Science at Department of Computer Science \& Electronics in Universitas Gadjah Mada, Yogyakarta, Indonesia (2018). She is working as a lecture in the department of Computer Science Universitas Mulawarman, Samarinda, Indonesia since 2009. Her research interest are image processing, pattern recognition and neural network. Email: anindita.septiarini@gmail.com 